\title{
Modernisierung der
}

\section{Versorgungsstrukturen in der Krankenversicherung}

KARL-HEINZ SCHÖNBACH, JÜRGEN MALZAHN

Karl-Heinz Schönbach ist Geschäftsführer Versorgung im AOK-Bundesverband, Berlin und Mitherausgeber der vorliegenden Zeitschrift

Jürgen Malzahn ist Leiter der Krankenhausabteilung im AOK-Bundesverband, Berlin

Deutschland stellt für alle Versicherten den Zugang zu einer modernen, hochstehenden und bedarfsgerechten Gesundheitsversorgung sicher. Diese Verlässlichkeit wäre nicht ohne eine gesellschaftliche Debatte möglich, in der Versorgungs-, Verteilungs- und Finanzierungsfragen als miteinander verbunden gesundheitspolitisch gelöst werden. Sie zu erhalten, stellt die Beteiligten immer wieder vor die Aufgabe, gesellschaftliche Veränderungsprozesse aufzunehmen und die funktionale Balance der Versorgungsstrukturen zu erhalten. Dies ist das Kontinuum der Reformpolitik, in Deutschland. Der Beitrag zeigt Kernpunkte des Reformbedarfs auf und gibt Hinweise, wie Fortschritte aus der bisherigen Systementwicklung heraus erreicht werden können.

\section{Ausgangslage}

In einer „Gesellschaft des langen Lebens" (SVR-G) ${ }^{1}$ treffen gerade die Gesundheitssysteme auf immense Herausforderungen: Während die Zahl älterer und temporär behandlungs- oder pflegebedürftiger Menschen demographisch zunimmt, wächst die Zahl nachrückender Beschäftigter in den Gesundheitsberufen nicht entsprechend. Auch wenn die sogenannte Kompressionsthese im Hinblick auf den Behandlungsbedarf in einer älter werdenden Gesellschaft geteilt wird und Zuwanderung in alle Berufen ein Gewinn sein wird, werden die Versorgungsstrukturen doch gefördert und effektiver und effizienter gestaltet werden müssen, um Angebot und Nachfrage immer wieder zu einem guten Ausgleich zu bringen. Dabei geht es für Deutschland im Kern darum, den in- ternationalen Rückstand in der Ambulantisierung ${ }^{2}$ der gesundheitlichen Versorgung auszugleichen und mit neuen, teamorientierten Versorgungsformen erfolgreich zu gestalten. Gerade dadurch werden Engpässe gemildert und Effizienzsteigerungen ermöglicht.

Diesen Prozess in den Mittelpunkt der Gesundheitspolitik zu stellen und sowohl im Leistungs- wie im Vertragsrecht der GKV zukunftsfest zu verankern, hat der Gesetzgeber bisher gezögert. Vielmehr sparen sowohl das

1 Vgl. SACHVERSTÄNDIGENRAT zur Begutachtung der Entwicklung im Gesundheitswesen: Koordination und Integration Gesundheitsversorgung in einer Gesellschaft des längeren Lebens, Sondergutachten 2009

2 Vgl. SACHVERSTÄNDIGENRAT zur Begutachtung der Entwicklung im Gesundheitswesen: Kooperation und Verantwortung Voraussetzungen einer zielorientierten Gesundheitsversorgung, Gutachten 2007, TZ 299 ff. 
öffentlich wenig beachtete Vertragsarztrechtsänderungsgesetz (VÄndG) 2007 wie das Versorgungsstrukturgesetz (VStG) 2012 mit seiner Spezialfachärztlichen Versorgung das Leistungs- und Vertragsrecht der GKV weithin aus $^{3}$. Die eingeleitete reale Flexibilisierung auf der Angebotsseite ist bisher ohne rechtliche Konsequenzen auf der Nachfrageseite geblieben: Eine übergreifende Bedarfsplanung, ein durchgängig patientenbezogenes Vergütungssystem und entsprechende individuelle Vertragsrechte ohne Kontrahierungszwang. Krankenkassen müssen jeden einzelnen Arzt unabhängig von seiner Spezialisierung und dem konkreten Bedarf als Partner innerhalb der Kassenärztlichen Vereinigung akzeptieren, obgleich er als Vertragsarzt gleichzeitig in einer Bandbreite zwischen „stationärer OP-Tätigkeit“ bis „privater Neben-Tätigkeit“ auch außerhalb der vertragsärztlichen Versorgung tätig sein kann. Das Leistungsgeschehen entzieht sich zunehmend den Verträgen. Es sind aber im Kern gerade die Verträge mit den Krankenkassen über in Entgeltverzeichnissen und Gebührenordnungen beschriebenen Leistungen, die das Versorgungsgeschehen immer wieder ausbalancieren. Nur wenn sie

\section{Entscheidend wird die reale Sicherstellung und nicht, ob wir uns Fehlallokation "monetär" leisten können.}

in einen hinreichenden ordnungspolitischen Kontext gestellt werden, kommen Erfolge der Gesundheitspolitik allen zugute: Patienten, Gesundheitsberufen und Beitragszahlern.

So hat etwa die Einführung des deutschen DRG-Systems mit diagnosebezogenen Fallpauschalen im Krankenhaus zweifellos einen deutlichen Produktivitätssprung mit sich gebracht, weil die Prozesse im Krankenhaus nun transparent organisiert werden können. ${ }^{4}$ Die Gesundheitspolitik hat es aber bisher versäumt, die notwendige Abkehr vom (Selbst-) Kostendeckungsprinzip mit einem System der leistungsbezogenen Krankenhausplanung abzusichern und das Vertragssystem für einen qualitätsorientierten Wettbewerb anzupassen: ${ }^{5}$
- Würden die Investitionskosten aber sinnvollerweise mit den Fallpauschalen entgolten und die Bundesländer eine bedarfsorientierte Raumplanung leisten, könnte die sich seit Jahren starre Unterauslastung der Krankenhäuser auflösen.

- Würden zudem Krankenhäuser und Krankenkassen spürbare einzelvertragliche Spielräume in einem Qualitätswettbewerb um planbare Leistungen haben, könnte die Patientensicherheit verbessert werden und innovative Krankenhäuser bei diesem Vergütungssystem stärker profitieren.

Die Unterauslastung hat sich aber als rigide erwiesen, obwohl insbesondere defizitäre Krankenhäuser - teils über die Grenzen medizinisch begründeter Indikationen hinaus - die „Flucht in die Menge" suchen. Offenbar ist das Rationalisierungspotential der Häuser im Durchschnitt immer noch größer als der expansive Leistungseffekt. Skandalöse Vorgänge in deutschen Krankenhäusern haben die Effekte fallorientierter Zielvereinbarungen für leitende Ärzte verdeutlicht. Da durch Leistungsvermehrung und Trägersubventionen hohe Überkapazitäten in der stationären Versorgung bleiben, werden dort im Ergebnis auch über den medizinischen Bedarf hinaus zusätzliche Ärzte gebunden. Diese Ärzte werden künftig dringend für die ambulante Versorgung gebraucht werden. Es geht mithin bei dem ordnungspolitischen Konzept für den stationären Sektor nicht länger darum, ob Deutschland sich eine duale Krankenhausfinanzierung mit Fehlallokation „monetär“ leisten kann, sondern um die Sicherstellung künftig „real“ fehlender Behandlungskapazitäten im ambulanten Sektor.

\section{Grundlegende Zielrichtung notwendiger Reformen}

Sowohl eine „Ambulantisierung“ der medizinischen Versorgung, die die Möglichkeiten und Chancen der Krankenhäuser einbezieht, als auch eine Stärkung der ambulanten vertragsärztlichen Versorgung durch Teamstrukturen und populationsbezogene Versorgungsaufträge ${ }^{7}$ werden im Mittelpunkt einer zu- kunftsgerichteten Gesundheitspolitik stehen müssen. Dabei gilt es im Kern, dem gesellschaftlich verantworteten Weg der GKV in hoher Kontinuität zu folgen und ebenso freiheitliche wie patientenorientierte Konzepte zu verwirklichen. Folgende sind Eckpunkte führend:

(1) Für den stationären Sektor sollte das gestufte System einer leistungsbezogenen und bedarfsorientierten Krankenhausplanung eingeführt werden ${ }^{8}$. Sie erlaubt den Ländern eine nach bundeseinheitlichen Grundsätzen regional angepasste Krankenhausplanung als Raumplanung und löst die teilweise enge und bettenbezogene Standortplanung ab. Diese Raunplanung wird unter Landesaufsicht von der Selbstverwaltung konkretisiert. Dafür scheint der Übergang zu einer monistischen Krankenhausfinanzierung notwendig, die zudem in den Leistungssegmenten einen relevanten Korridor für die Vermeidung von Kontrahierungszwang beinhaltet, wo dies Gesichtspunkte von Patientenschutz und Qualitätssicherung dringend gebieten. Dieser Vorbehalt

3 Vgl. Schönbach, K.H., Malzahn, J., Wehner, Chr.: Sicherstellungsfrage neu gestellt Chancen für Direkterträge in der GKV, in Gesundheits-und Sozialpolitik, 5 in 2010, S. 10. ff.

4 Vgl. IGES: DRG-Begleitforschung gemöß $\S 17 \mathrm{~b}$ Abs. $8 \mathrm{KHG}$ - Endbericht des dritten Forschungszyklus (2008 bis 2010, Untersuchung im Auftrag des deutschen DRGInstituts (InEK), Berlin, März 2013.

5 Malzahn, Jürgen; Wehner, Christian: Zur Lage der Investitionsfinanzierung der Krankenhäuser - Bestandsaufnahme und Reformbedarf; in: Klauber, Jürgen; Geraedts, Max; Friedrich, Jörg (Hrsg.): Krankenhaus-Report 2010 - Schwerpunkt: Krankenhausversorgung in der Krise, Stuttgart 2010, S. 107-125.

6 Vgl. Mohrmann, Matthias; Koch, Volker: Selektivverträge im Krankenhausbereich als Instrument zur Verbesserung von Qualität und Effizienz; in: Klauber, Jürgen; Geraedts, Max; Friedrich Jörg; Wasem, Jürgen (Hrsg.): Krankenhaus-Report 2011 - Schwerpunkt: Qualität durch Wettbewerb, Stuttgart 2011, S. 61-79.

7 Entsprechenden Reformbedarf greift der Bericht der Böll-Stiftung auf: „Wie geht es uns morgen? Wege zu mehr Gesundheit und Gesundheitsnutzen in einem solidarischen Gesundheitswesen“, Bericht der Gesundheitspolitischen Kommission der Heinrich-Böll-Stiftung : Mehr Gesundheitseffizienz: Von der Kranken- zur Gesundheitsversicherung - neue Anreiz- und Steuerungsstrukturen im Gesundheitswesen, Berlin, Juni 2013.

8 Vgl. Karl-Heinz Schönbach, Hans-Georg Faust: Von einer planlosen Überversorgung zur sektorenübergreifenden Versorgungsplanung? In: Gesundheits- und Sozialpolitik, Ausgabe 4 in 2012, Seite $8 \mathrm{ff}$. 
gilt gerade planbaren Eingriffen mit extensiv auszulegenden Indikationsgrenzen, besonderen Risiken bei der Ergebnisqualität und umstrittenem Patientennutzen.

(2) Der schrittweise Aufbau einer erweiterten, sektorenunabhängigen spezialärztlichen Versorgung, in der die ambulante Beteiligung der Krankenhäuser neu geordnet und mit den entsprechenden vertragsärztlichen Angeboten wettbewerbsgleich in ein Vertragssystem geführt wird. Dafür scheint der schrittweise Aufbau einer übergreifenden Bedarfsplanung ebenso erforderlich wie ein durchgehend patientenorientiertes, d.h. indikationsbezogenes Vergütungssystem und ordnungspolitische Vorgaben für einen Qualitätswettbewerb, der den Patienten einen möglichst weiten Zugang zu ärztlichen Spezialisten sichert, ohne Einrichtungen mit offensichtlichen Qualitätsmängeln vertraglich einzuschließen.

(3) Für den ambulanten Sektor ist eine notwendige Bedingung ein modernes, weil „nachfragesensitives und lernfähiges“ Vergütungssystem ${ }^{9}$ für die ambulante ärztliche Versorgung. Indikationsbezogene Fallpauschalen bieten auch ambulant gute Chancen, Patientenorientierung der Leistungen, Therapiefreiheit des Arztes, begleitende Qualitätssicherung und die Nutzung von Teamstrukturen mit einer angemessenen Finanzierung zu verbinden. Zudem müssen Leistungen vermehrt am gesundheitlichen Problem des Patienten ausgerichtet, hinreichend komplex konzipiert und vergütet werden, damit darauf aufbauend für die Patienten entsprechende ambulante Versorgungsstrukturen entstehen können und stationäre Behandlung substituiert werden kann.

Diese Ziele ergeben sich aus der systemimmanenten Kontinuität der deutschen Reformpolitik im Gesundheitswesen und ergänzen die eingeleitete Flexibilisierung auf der Angebotsseite um adäquate Impulse auf der Nachfrageseite, ohne die sich die Versorgung nicht an veränderte Bedürfnisstrukturen einer alternden Gesellschaft anpassen kann.

Wird an dieser Stelle der Reformbedarf der Versorgungsstrukturen be- tont, können die Finanzierungsfragen gleichwohl nicht als weniger drängend gelten. Der Wissenschaftliche Beirat für den RSA beim Bundesversicherungsamt (BVA) und das BVA selbst haben keinen Zweifel gelassen, dass der Methodenfehler des M-RSA korrigiert werden muss, inzwischen wurde dies in einem ersten Urteil bestätigt ${ }^{10}$. Allerdings wird man für die Finanzierungsseite im Ergebnis komplexer politischer Abwägungen davon auszugehen haben, dass

(1) es für die Finanzierung der Krankenversicherung innerhalb des Gesundheitsfonds auch künftig bei lohnund lohnersatzbezogenen Beiträgen bleiben wird,

(2) die besonderen Lasten aus der demographischen Entwicklung eine nachhaltige Heranziehung der übrigen Einkunftsarten durch allgemeine steuerfinanzierte Einträge in den Gesundheitsfonds erfordern und

(3) somit allein aufgrund der notwendigen allgemeinen Steuerfinanzierung der GKV eine Rückkehr zur Beitragssatzautonomie auf der Ebene der einzelnen Krankenkasse erschwert ist.

Gesetzlich ohne Wenn und Aber sicherzustellen ist eine hinreichend hohe Mittelausstattung des Gesundheitsfonds, so dass sozial abgefederte Zusatzbeiträge ein politischer Grenzfall bleiben. Im Interesse eines regional und sozial für alle Versicherten bedarfsgerechten Zugangs zur gesundheitlichen Versorgung muss die größte Aufmerksamkeit vielmehr auf der einen Seite dem Ausgleich von Risiko- und Finanzierungsstrukturen der Krankenkassen gelten und auf der anderen Seite den erforderlichen Rahmenbedingungen und Anreizen, die Effektivität und Effizienz des beitrags- und steuerfinanzierten Mitteleinsatzes zu gewährleisten.

\section{Treibende Entwicklungen}

\section{Demographische Änderungen der Nachfrage}

Die Bevölkerungsprognosen des Statistischen Bundesamtes (StBA) sind vielfach publiziert. In der anstehenden Zeitspanne von 2015 bis 2050 wird sich der
„Altersquotient 65“ der Bevölkerung in Deutschland beinahe verdoppeln; der „Altersquotient 85 “ wird sich rund verdreifachen. Bereits bis 2025 wird sich ein spürbarer Bevölkerungsrückgang vollziehen, der vor allem Ost-Deutschland und dort insbesondere die ländlichen Räume betrifft. Welcher Teil dieser Entwicklung von Zuwanderung abgeschwächt wird, muss in den Prognosen offen bleiben. Bereits bezifferbar abzuschätzen ist jedoch, dass sich allein die Zahl der Pflegebedürftigen von zurzeit rund 2,2 Mio. bis zum Jahre 2040 auf 3 bis 3,5 Mio. Personen erhöhen wird. Über den zunehmenden Pflegebedarf hinaus wird sich angesichts der Alterssteilheit der Ausgaben für medizinische Leistungen ein zusätzlicher Behandlungsbedarf ergeben. Auch wenn die zahlreich vorliegenden Schätzungen der davon ausgehenden finanzwirtschaftlichen Beitragssatzeffekte kompensatorische Entwicklungen kaum hinreichend berücksichtigen können, steigt der Pflege- und Behandlungsbedarf doch real an: Der Anteil von Menschen mit chronischen, altersbedingten und Vielfacherkrankungen sowie Pflegebedürftigkeit wird zunehmen, selbst wenn sich ein größerer Teil der Prävention verhaltensbedingter Erkrankungen als erfolgreich erweist.

\section{Die Versorgung multimorbider Patienten und geriatrische Leistungen werden einen höheren Stellenwert erlangen.}

Die Versorgung multimorbider Patienten und geriatrische Leistungen einschließlich der Rehabilitation und Pflege werden einen höheren Stellenwert erlangen. Diese Leistungen sind regelmäßig personengebunden, zuwendungsintensiv und nicht rationalisierbar. Sie stehen im Kern des Leistungsversprechens der Generationen. Insbesondere die Finanzierung medizinisch-technischer Innovationen, soweit von ihnen kein Ra-

9 Entsprechenden Reformbedarf greift der Bericht der Ebert-Stiftung auf: Wettbewerb, Sicherstellung und Honorierung: Neuordnung der Versorgung im deutschen Gesundheitswesen, Positionspapier im Auftrag der Abt. Wirtschafts- und Sozialpolitik der Friedrich-Ebert-Stiftung, April 2013.

$10 \mathrm{Vgl}$. WAZ vom 05.07.13, Seite 4, Gericht kippt Finanzausgleich der Kassen 
tionalisierungspotential ausgeht, muss mit überprüfbarem Kosten-NutzenMaßstab dagegen aufgewogen werden.

\section{Demographische Änderungen des Angebots}

Dem tendenziell steigenden Behandlungsbedarf steht ein in den kommenden Jahrzehnten zunächst im günstigen Fall gleich bleibendes Behandlungsangebot gegenüber. Daraus lässt sich unter sonst gleichen Bedingungen eine Angebotslücke beziffern, die angesichts der hohen Attraktivität des Arztberufes in besonderem Maße für nichtärztliche Heilberufe angenommen wird. Im Hinblick auf ärztliche Kapazitäten scheinen die Probleme in der Schweiz, Großbritannien und die skandinavischen Länder ungleich größer, die heute deutlich unter ihrem eigenen aktuellen Bedarf ausbilden. Demgegenüber wird in Deutschland, das bevölkerungsbezogen bereits heute mehr Ärzte ausbildet und beschäftigt als jedes andere Land, vor allem die Produktivität je Arzt (-Stunde) durch eine bedarfsgerechte Allokation der Ärzte und Sitze sowie durch eine teamorientierte Leistungserbringung und zielgerichtete Umgestaltung der Vergütungssysteme zu erhöhen sein: Es müssen nicht über Apelle und „Bedarfsplanung“, sondern über konkret formulierte Leistungen und Nachfrage und Finanzierung

- relativ mehr Ärzte als bisher in der hausärztlichen Versorgung tätig werden,

- relativ mehr Ärzte als bisher in ländlichen Räumen tätig werden,

n höhere Investitionen in die ambulante Infrastruktur und in die Arbeitsteilung einerseits der ärztlichen Gebiete und andererseits der ärztlichen und nichtärztlichen Heilberufe gelenkt werden,

- strukturelle Hemmnisse wie Quartalsbezüge in Abrechnungssystemen und „gelbe Scheine“ kritisch diskutiert werden.

In den letzten Jahrzehnten hat sich die Relation „Arzt zu nichtärztlichen Heilberufen“ in der Versorgung - unabhängig von einer primär staatlichen oder einzelwirtschaftlichen Organisationsform - sowohl in Großbritannien als auch in den USA oder Skandinavien deutlich zugunsten der Nichtärzte verschoben, während sie in Deutschland vergleichsweise starr blieb. Diese auch aus der (unternehmens-) rechtlichen Arztzentrierung des deutschen Gesundheitswesens und entsprechenden Grundsätzen der Leistungserbringung und Honorierung resultierende Beschränkung:

- behindert teamintegrierende Angebotsformen,

- verzichtet auf Produktivitätsgewinne für ärztliche Leistungen

- und schränkt den professionellen Spielraum nichtärztlicher Berufe ein.

Ansätze zumindest für eine

Delegation im vertragsärztlichen Bereich (AGNES, VERAH) blieben - ausgehend von den neuen Bundesländern und Hausarztverträgen - zunächst auf Modelle und nach Maßstäben der Bedarfsplanung unterversorgte Gebiete beschränkt. Fachspezialisierte „Nurses“ nach angelsächsischem und skandinavischem Vorbild würden erhebliche Angebotsverbesserungen mit sich bringen. Generell sollte im pflegerischen Bereich eine Aufwertung von Ausbildung, differenzierter Qualifikation, Kompetenzzuweisung und Verantwortung die Attraktivität des Berufes erfolgen, um die Verweildauer im Beruf der dort Tätigen maßgeblich erhöhen.

\section{Spezialisierung und Kooperationsbedarf}

Es ist ohne weiteres nachzuvollziehen, dass die berufsständische Selbstverwaltung der Ärzte im Binnenverhältnis in hohem Maße autonom die professionelle Anerkennung nach der Spezialisierung und besonderen Leistungsfähigkeit differenziert. Immer mehr ärztliche Spezialisierungen und Subspezialisierungen sind die Folge. Im Außenverhältnis zu den Vertragspartnern und wirtschaftlich darf die „autonome“ Binnenlogik allerdings nicht unkorrigiert bleiben. An dieser Stelle politisch zu resignieren und der Kassenärztlichen Vereinigung über ihr Vertragsrecht hinaus auch noch das Verteilungsmonopol der Honorare zuzuweisen ist allokativer Nihilismus: Die Politik erhebt damit die jeweiligen Stimmenverhältnisse in Beschlussgremien der Kassenärztlichen Vereinigung zur „letzten Instanz“ für die Mittelverteilung in der ambulanten ärztlichen Versorgung. Im
Gegenteil sollten für den „Einheitlichen Bewertungsmaßstab ärztlicher Leistungen (EBM) nachfrageseitige Öffnungen gewährleistet sein. Es kann nicht angehen, dass sich „Subspezialisten“ mit ebenso aufwendigem wie teurem Equipment in immer höherer Zahl niederlassen, um wenige Patienten zu behandeln,

Kassenärztlichen Vereinigung das Verteilungsmonopol der Honorare zu überlassen, ist llokativer Nihilismus.

während etwa

- einerseits für chronisch kranke, psychiatrische und geriatrische Patienten ausreichende Angebote in qualifizierten Teamstrukturen fehlen (Schwerpunktpraxen und interdisziplinäre Netze)

- und andererseits die Versorgung von akut schwerwiegenden Erkrankungen und komplexe Eingriffe ambulant nicht auf besonders erfahrene und qualitätsgesicherte Zentren konzentriert werden kann.

Etwa mit onkologischen Schwerpunktpraxen und ihren Vernetzungen ist der Einstieg erbracht, dass ambulante Behandlung bei schwerwiegenden Erkrankungen qualitativ koordiniert sichergestellt werden kann. Hierzu haben Berufsverband, KBV, Krankenkassen und Krebshilfe zusammengearbeitet. In weiten Teilen der ambulanten Versorgung fehlen aber entsprechende Initiativen. Angesichts der defizitären Budgetbereinigung gerade für fachärztliche Leistungen kann auch nicht darauf gesetzt werden, dass allein einzelvertragliche Initiativen der Krankenkassen eine qualitative Strukturierung ambulant hinreichend vorantreiben können. Auch wenn Einzelverträgen eine besondere Innovationsfunktion zukommt, müssen auch die kollektivvertraglichen Partner in der Lage und dazu bereit sein, etwa indikationsbezogene Leistungsbündel, ihre Voraussetzungen und Behandlungspfade zu vereinbaren. Der Gesetzgeber hat im VStG die Richtung dazu mit der Spezialfachärztlichen Versorgung gewiesen, ohne dafür ein vertragspartnerschaftlich tragfähiges und reproduzierbares 
Konzept bereitzustellen. Der weiteren Entwicklung dieses Konzepts sollte ausgehend von der Schnittstelle ,ambulantstationär" größtes Gewicht zukommen.

\section{Mangel an Effektivität und Effizienz der Mittelverwendung}

Vom Grundsatz her ist der Effektivität der Versorgung am besten gedient, wenn Therapiefreiheit sichergestellt ist und die Ärzte systematisch voneinander lernen. Dies setzt die Kenntnis der Standards, des Leistungsgeschehens selbst und angemessene Qualitätssicherung voraus. Effizienz verlangt den produktiven Einsatz der Mittel. In hohem Maße infrage gestellt sind diese Ziele jedoch, wenn eine Gebührenordnung, die diese Anforderungen unterstützen soll, systematisch „verrichtungsbezogen“ statt „patientenbezogen“ formuliert ist und keine fortlaufend aktualisierten Maßstäbe für den regelmäßig erforderlichen Mitteleinsatz kennt. Ärztliche Verrichtungen sind deutlich begründungsärmer zu vermehren als patientenbezogene Behandlungsanlässe. Und da die wenigsten
Vergütungen ambulant realistisch mengenbezogen kostenkalkuliert sind, ist der Fehlallokation Tür und Tor geöffnet:

- So ist die Zahl der Arzt-PatientenKontakte in Deutschland mit durchschnittlich 18 Kontakten pro Jahr ohne Vergleich in der internationalen Statistik.

- Aufwendige und für den Patienten risikoreiche Verfahren wie Herzkatheter-Untersuchungen erreichen in Deutschland allein doppelt so hohe Frequenzen wie in vergleichbaren Länder wie der Schweiz.

- Die Häufigkeit bildgebender Verfahren an deutschen Kliniken ist allein von 2006 bis 2009 um 52 Prozent angestiegen.

- Und unter den ambulanten Eingriffen nehmen etwa Arthroskopien der Gelenke gerade dort mit hohen Steigerungsraten $\mathrm{zu}$, wo die Indikation dazu regelmäßig nicht gegeben ist.

Nur ein erschreckend geringer Teil dieser Mehrleistungen ist auf die demographische bzw. morbiditätsbedingte

\section{Abbildung 1: Entwicklung der Linksherzkatheter-Untersuchungen von 1990 bis 2009 und Sterbeziffern nach Herzinfarkt im 3-Länder-Vergleich.}
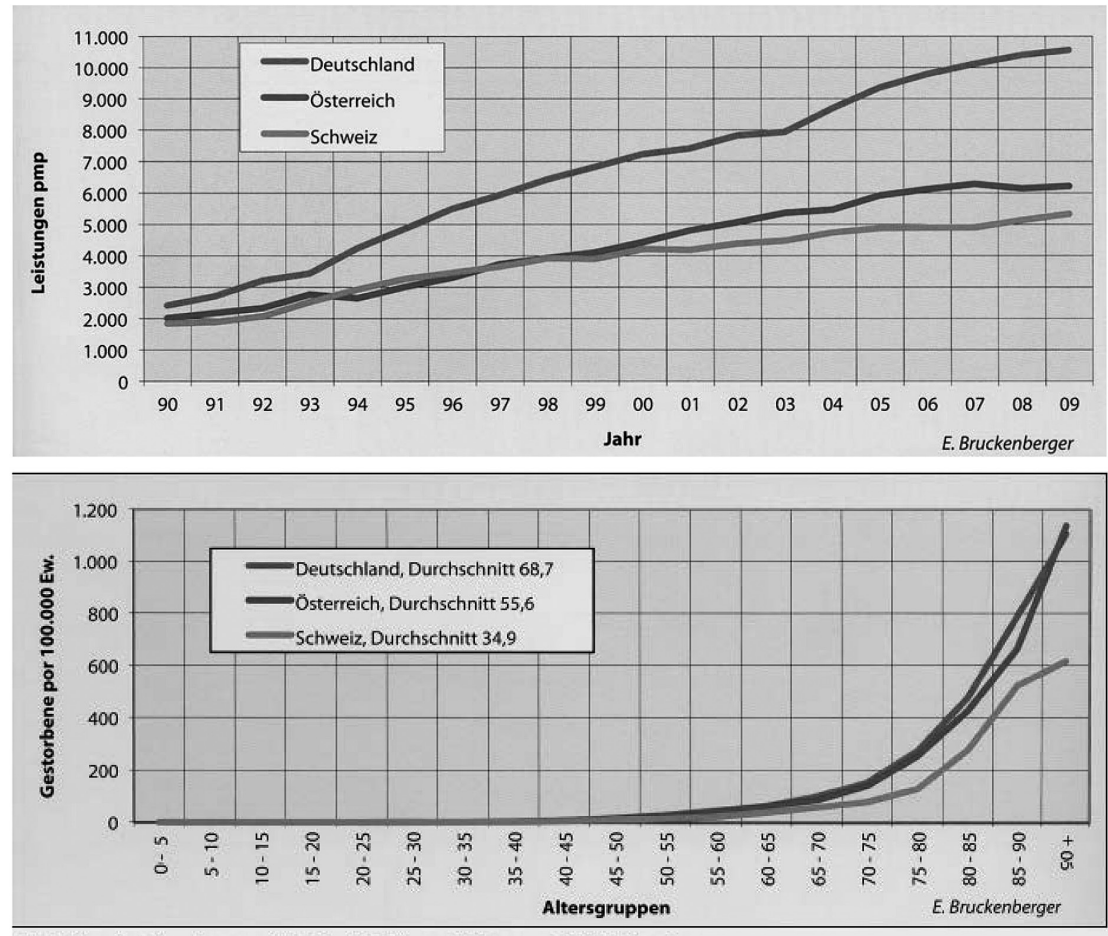

-Sterbeiffer = Gestarbene bezogen auf die Gräße der Bevöikerung, hier berogen ouf 100.000 Eirwohner

Quelle: Bruckenberger, E.: 22. Herzbericht 2009; 17. Bericht Sektorenübergreifende Versorgungsanalyse zur Kardiologie und Herzchirurgie in Deutschland: Mit vergleichenden Daten aus Österreich und der Schweiz; Hannover 2010.
Entwicklung zurückzuführen. Ebenso steht außer Frage, dass der begrüßte medizinische Fortschritt diese Dynamik in keiner Weise erklärt. Vielmehr wirken an dieser Stelle (zu Recht) leistungsbezogene Vergütungssysteme, die bisher weder durch hinreichende Qualitätsvereinbarungen noch einzelvertragliche Absprachen ausbalanciert sind. Regionale Gesamtvertragspartner können diese Entwicklung meist nur beobachten, Daten für lange nachgehende Auswertungen sammeln oder globale finanzielle Begrenzungen treffen. Notwendig wären stattdessen angemessen differenzierte, mit Parametern der Ergebnisqualität ausgestattete und eine Qualitätssicherung mit longitudinalen Routinedaten aufgreifende Vereinbarungen. An dieser Stelle besteht dringender Reformbedarf, ausgehend allein schon von der nicht nachvollziehbaren Aufhebung der Diagnosekodierung des Vertragsarztes mit dem VStG.

Insgesamt wird an vielen Stellen deutlich, dass Mitteleinsatz und Untersuchungshäufigkeiten keinen zusätzlichen Ertrag an Gesundheit für die Versicherten mit sich bringen, sondern primär nicht ausbalancierten ökonomischen Anreizen folgen. Die nachstehende Abbildung illustriert, dass etwa die Zunahme der Herzkatheter-Leistungen in Deutschland der Entwicklung in der Schweiz und Österreich vorauseilt, ohne dass dies positive Effekte bei der Veränderung der Sterberate hätte. Der Nutzen für die Patienten wird nicht durch noch „mehr“ Diagnostik verbessert werden, sondern gerade durch eine gute Vor-und Nachsorge im ambulanten Bereich etwa in Verbindung mit der guten Einstellung des Blutdrucks. Auch hier wird deutlich, dass ein immer höherer Mitteleinsatz und zunehmende Untersuchungshäufigkeiten keinen zusätzlichen Ertrag an Gesundheit für die Versicherten mit sich bringen, sondern häufig falschen, nicht ausbalancierten ökonomischen Anreizen des Gesundheitswesens geschuldet sind.

\section{Sicherstellung der ambulanten Versorgung}

\section{a. Versorgungsstrukturen}

\section{Hausärztliche Versorgung}

Die hausärztliche Versorgung als Rückgrat des ambulanten Systems leidet seit vielen Jahren am unzureichenden Aus- 
bau allgemeinmedizinischer Lehrstühle, Engpässen in Teilen der Weiterbildung und im Hinblick auf die umfassende Versorgungsaufgabe der Hausärzte unterkomplexe Versorgungsstrukturen. So nimmt nicht nur der Anteil der Hausärzte an der Gesamtzahl der ambulant tätigen Ärzte ab, vielmehr bleibt die Zunahme hausärztlicher Praxisgemeinschaften deutlich hinter der Kooperation fachärztlicher Gebiete zurück. Offenbar stehen Synergien wie die gemeinsame Gerätenutzung vor einer versorgungsinhaltlichen Kooperation. Bleibt der Hausarzt in der KV aber „Einzelkämpfer“, obgleich er die bei weitem vielfältigste Versorgungs- und Verknüpfungsaufgabe wahrzunehmen hat, erweisen sich Hausarztverträge als umso unverzichtbarer. Sie sichern den Hausärzten über Qualitätszirkel hinaus medizinische und ökonomische Unterstützung, Vernetzung und Erfolgsbestätigung.

\section{Förderung einer fachärztlichen Grundversorgung}

Zwischen den vitalen hausärztlichen Funktionen und dem Kern der fachärztlichen Kompetenzen in der spezialisierten Medizin verbleibt eine fachärztliche Grundversorgung, die wie die hausärztliche Ebene wohnortnahe Leistungen erbringt. Eine Förderung dieser Grundversorgung sollte auf den essentiellen Kern konzentriert werden, der weder der hausärztlichen Grundversorgung zuzurechnen ist, noch den im Kern fachärztlich spezialisierten Leistungen. Darüber hinaus konserviert der Erhalt einer „doppelten Grundversorgung“ in erster Linie historisch bereits relativierte Versorgungsstrukturen. Vielmehr muss die Förderung von Leistungen im Kern dem Patientennutzen folgen.

\section{Delegation und Substitution ärztlicher Leistungen}

An den Grenzen der hausärztlichen Unterversorgung wird bisher schon der Einsatz von besonders fortgebildeten medizinischen Fachhelferinnen in der Häuslichkeit des Patienten ermöglicht. Die Leistungen finden insbesondere aufgrund der besonderen Fachlichkeit und Zuwendung herausgehobene Zustimmung bei den Patienten. Daher soll auf der Grundlage einer G-BA-Richtlinie auch die Substitution bestimmter ärzt- licher Tätigkeiten durch qualifizierte Pflegekräfte erprobt werden. Die Krankenkassen hoffen auf positive Erfahrungen und sehen in Modellversuchen nicht zuletzt einen positiven Beitrag, medizinische Teamstrukturen zu entwickeln. Eine Verkammerung der Pflegeberufe könnte Motor für eine differenzierte Qualifizierung sein, die begrüßt wird, auch wenn die Form selbst wenig zukunftsgerichtet scheint.

\section{Besondere Versorgungsformen}

Während sich die Hausarztzentrierte Versorgung $(\mathrm{HzV} ; \mathbb{S} 73 \mathrm{~b}$ SGB V) auf der Grundlage einer funktionsfähigen Budgetbereinigung in weiten Teilen der GKV zu einer flächendeckenden Alternative kollektivvertraglich vereinbarter ambulanter Sicherstellung entwickelt hat, ist die Integrierte Versorgung (IV, $\mathbb{S} 140 \mathrm{~b}$ SGB V) indikationsbezogen auf besondere regionale Versorgungsaufgaben gerichtet. Davon zu unterscheiden sind populationsbezogene Versorgungsformen wie sie etwa in Arztnetzten eine Ausprägung gefunden haben. Anders als etwa in der Schweiz, wo es keine Kassenärztlichen Vereinigungen gibt, ist die Bereinigungsfähigkeit der prospektiv vereinbarten Gesamtvergütung ein Nadelöhr für die Weiterentwicklung moderner Organisationsformen der Leistungserbringung. Es ist dringend geboten, freie, nicht von Tendenzbetrieben der verfassten Ärzteund Apothekerschaft beherrschte Infrastrukturen für die Kommunikation und das Abrechnungsgeschäft besonderer Versorgungsformen zu etablieren und Blockademöglichkeiten abzubauen, mit denen der personalintensivste und dynamischste Wirtschaftszweig seiner möglichen Kooperationsinfrastruktur weithin um Jahrzehnte zurückgeworfen wird.

\section{Bereinigung der Gesamtvergütungen}

Bisher gilt das Modernisierungsparadigma, kollektive Flächenverträge durch einzelvertragliche Alternativen zu öffnen und aus der Kollektivvergütung $\mathrm{zu}$ bereinigen. Das Grundprinzip der Bereinigung auf Basis der historischen Leistungsmengen ist einfach, verständlich und sachgerecht. Es erfüllt auch die gesetzliche Bedingung der Risikoadjustierung. Das Verfahren kann von den Vertragspartnern selbst umgesetzt wer- den; wissenschaftliche Expertise oder ein „Bereinigungsinstitut“ sind nicht erforderlich. Anfangsfragen werden vor den Schiedsämtern gelöst. Insoweit hat sich das Bereinigungsverfahren bereits für die hausarztzentrierte Versorgung angesichts der Rahmenbedingungen Primat der Kollektivversorgung und überaus ablebnende Haltungen der Kassenärztlichen Vereinigungen - in der Praxis bewährt. Dennoch wird eine wettbewerbsneutrale Bereinigung „grundständig“ behindert,

- weil die gesetzlichen Regelungen zur Bestimmung der „morbiditätsbedingten“ Gesamtvergütung lediglich die Seite der Mittelaufbringung bei der Kassenärztlichen Vereinigung adressieren, während die Morbidität bei der Honorarverteilung lediglich ein von zahlreichen anderen Variablen überlagerter Faktor ist und

n weil die Honorarverteilung zudem mit dem GKV-VStG erneut ausschließlich durch die KVen gestaltet wird, wodurch Wettbewerbsverzerrungen entstehen, wenn etwa aufgrund der (risikoadjustierten) Bereinigung von besonders kranken Versicherten, bei dem betroffenen Arzt mehr bereinigt wird, als dieser in seinem (nicht risikoadjustierten) Regelleistungsvolumen bzw. in seinem erwarteten Honorar für diesen Versicherten zugewiesen bekäme.

Um die Verzerrungen zwischen (Gesamt-)Vergütung und Honorar zu vermeiden, wäre eine rational medizinisch begründete, indikationsbezogen aufwandsgerechte und über die Sektoren durchgängige Leistungs- und Vergütungsdefinition, die sowohl die Vergütung durch die Krankenkassen als auch die Honorierung der Ärzte konsistent abbilden würde, erforderlich.

\section{Beteiligung der Krankenhäuser an der ambulanten Versorgung}

Die ambulante Behandlung in Krankenhäusern weiterhin systematisch als „Ausnahme von der Regel“ getrennter Versorgungs- und Vergütungsbereiche zu betrachten verbietet bereits der Blick in die Versorgungswirklichkeit. Rund die Hälfte der Patienten, die heute mit dem Krankenhaus in Berührung kommen, erhält keine Behandlung „am 
Bett". Formen und Anlässe der ambulanten Behandlung im Krankenhaus wurden über Jahrzehnte jeweils als neue „Ausnahme“ geschaffen, ohne dass zumindest im Nachhinein ein stimmiges Konzept hinterlegt worden wäre. ${ }^{11}$ Von der Bedarfsplanungs-, Vertrags-, Vergütungs-, Mengensteuerungs- und Qualitätssicherungsseite her ist der entstandene Flickenteppich konzeptionell neu als „sektorenunabhängige Versorgung“ zu ordnen. Heute bestehen nebeneinander:

- Vor- und nachstationäre Behandlung im Krankenhaus ( $\$$ 115a SGB V),

- teilstationäre Behandlung im Krankenhaus (\$ 39 SGB V),

- die Hochschulambulanzen ( $\mathbb{S} 117$ SGB V),

- das ambulante Operieren $(\mathbb{S} 115 \mathrm{~b}$ SGB V),

- die Behandlung von seltenen Erkrankungen und Krankheiten mit besonderem Krankheitsverlauf ( $\mathbb{S} 116 \mathrm{~b}$ SGB V),

- die psychiatrischen Institutsambulanzen ( $\mathbb{S} 118$ Abs. 2 SGB V),

- die Sozialpädiatrischen Zentren (\$ 119 SGB V),

- die pädiatrischen Spezialambulanzen ( $\int 120$ Abs. 1a SGB V),

- ambulante Ermächtigungen von Krankenhausärzten (\$116 ff. SGB V)

- die psychiatrischen Institutsambulanzen an psychosomatischen Einrichtungen ( $\mathbb{S} 118$ Abs. 3 SGB V)

- geriatrischen Institutsambulanzen ( $\int 118$ a SGB V)

- und ambulante Versorgung bei Unterversorgung ( $\mathbb{1} 116 \mathrm{a}$ SGB V).

\section{b. Sektorenunabhängige ambulante Versorgung}

Die Formen der ambulanten Behandlung im Krankenhaus sollten schrittweise überprüft und systematisch neu in das Vertragsrecht der GKV eingeordnet werden. Dabei besteht jeweils die Chance, den jeweiligen ambulanten Versorgungsauftrag des Krankenhauses nach dem Vorbild der Spezialfachärztlichen Versorgung unter Einbezug der entsprechenden vertragsärztlichen Leistungen wettbewerbsgleich zu einer sektorenunabhängigen Versorgung zu verbinden. An die Stelle kollektiver Bewirtschaftung nach primär finanzwirtschaftlichen Begrenzungsregelungen tre-

\section{Abbildung 2: Einordnung einer sektorenunabhängigen Versorgung}

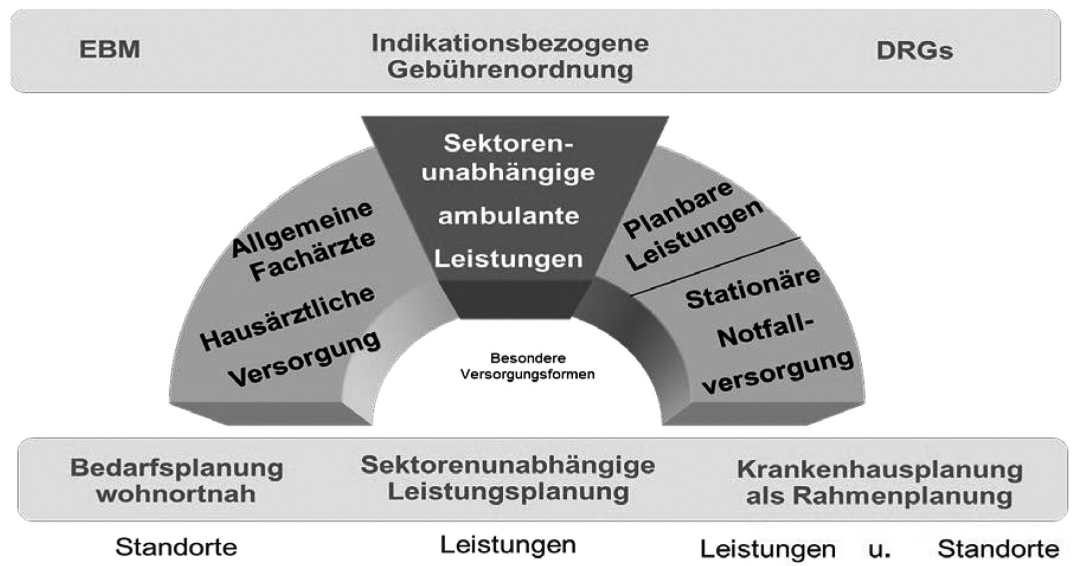

ten an dieser Stelle offener Zugang für die Patienten, Qualitätssicherung und Qualitätswettbewerb unter Einschluss aufsichtsrechtlich geschützter Wettbewerbsregeln. Für die allgemeinen Versorgungsformen zeigt Abb. 2 eine Einordnung.

- Danach verblieben die hausärztliche und allgemeine fachärztliche Versorgung (fachärztliche Grundversorgung) in der Obhut der ambulanten Bedarfsplanung und der Finanzierung nach Prinzipien der vertragsärztlichen Vergütung. Die Bedarfsplanung erfolgt an dieser Stelle wohnortnah nach Arztsitzen. Die hausärztliche Honorierung wird weiter innerhalb einer separaten Gesamtvergütung regelmäßig nach Fallpauschalen und Zuschlägen insbesondere für chronische Erkrankungen zu gestalten sein. Bei Abstaffelungsgrenzen für zusätzliche Einzelleistungen sollte die durchschnittliche Morbidität der Praxis berücksichtigt werden.

- Die stationären Leistungen, die notwendigerweise unter Zuhilfenahme des Bettes zu erbringen sind, verblieben in der Krankenhaus(raum!) planung unter der Letztverantwortung bzw. Aufsicht der Länder und unter Finanzierung der DRGs. Auf den Reformbedarf der Krankenhausplanung, den schrittweisen Übergang zu einer monistischen Krankenhausfinanzierung und eine neue Architektur der Vertragsrechte wird in Kapitel 8 einzugehen sein.

- Und die Leistungen der „sektorenunabhängigen Versorgung “ werden nach einer neuen, schrittweise aufzubauenden „patientenbezogenen“ Gebührenordnung vergütet. In der dafür vorzusehenden sektorenübergreifenden Versorgungsplanung wird die Verfügbarkeit von Leistungen sektorenunabhängig geplant. Um Qualitätsvorteile und Skaleneffekte nutzen zu können, entstehen in diesem Bereich zunehmend Zentren und Qualitätsnetze wie in der Onkologie und anderen Gebieten bereits der Zielstandard.

Auf diese Weise können über die vertragsärztliche Versorgung hinaus fachärztliche Kapazitäten der Krankenhäuser systematisch in die ambulante Behandlung einbezogen werden. Ob, wann und inwieweit sie in die ambulante Behandlung einbezogen werden, muss Gegenstand der ambulanten bzw. sektorenübergreifenden Bedarfsplanung sein. Allein von daher verbieten sich Konstruktionen sektorenübergreifender Gremien etwa nach $\int 90$ a SGB V, bei denen die (auch) ökonomisch verantwortlichen Partner nicht in Parität gegenüberstehen und das Land nicht ohne Beeinträchtigung seiner Aufsichtsfunktion nachkommen kann. Anhörungsrechte Dritter bleiben davon unberührt.

Eine sektorenübergreifende Bedarfsplanung wird sich künftig auf einen gleichnamigen, indikationsbezogenen Leistungsbegriff beziehen müssen, wie er

11 Vgl. Leber, Wulf-Dietrich; Klein-Hitpaß, Uwe: Spezialärztliche Versorgung - Plädoyer für eine Neuordnung; in: Klauber, Jürgen; Geraedts, Max; Friedrich, Jörg (Hrsg.): Krankenhaus-Report 2012 - Schwerpunkt: Regionalität, Stuttgart 2012, S. 205-236. 
mit einer neuen ambulanten Gebührenordnung entsteht. Insoweit ist die geforderte indikationsbezogene Gebührenordnung ein konstitutiv notwendiges Instrument der Sicherstellung. Es bedarf dann auf entsprechend sektorenunabhängiger empirischer Grundlage bzw. Grundlage einer Versorgungsberichterstattung angemessener Richtlinien des Gemeinsamen Bundesausschusses (G-BA) zu einer sektorenunabhängigen Bedarfsplanung. Dabei sind naturgemäß schrittweise dort ambulante Krankenhauskapazitäten systematisch einzubeziehen, wo die ambulante Versorgung durch Vertragsärzte unterhalb der durchschnittlichen Bedarfsgrade bleibt. Eine in dieser Weise ausgestaltete „sektorenübergreifende Bedarfsplanung " lässt sich harmonisch in bestehende Strukturen einpassen.

\section{Weiterentwicklung der Spezial(fach)ärztlichen Versorgung}

Aktuell hat der SVR-G Empfehlungen zum Wettbewerb an der Schnittstelle zwischen ambulanter und stationärer Versorgung abgegeben, die ebenfalls Wege ausgehend von der „Spezialfachärztlichen" Versorgung beschreiben, nämlich die oben angeführte schrittweise Neuordnung der ambulanten Behandlung im Krankenhaus in Verbindung mit einer sektorenunabhängigen diagnosebezogenen Gebührenordnung. Im Kern formuliert der SVR-G das Entwicklungskonzept für einen sektorenunabhängigen Versorgungsbereich mit „Vertragspflicht“ der Krankenkassen" ${ }^{12}$ : „Ein sektorenübergreifender Wettbewerbsansatz, der die Sicherstellung der ambulanten Versorgung garantiert, die ambulanten Krankenhausleistungen einbezieht und kalkulierbare Vergütungen sichert, ist möglich. Statt des Kontrahierungszwangs mit jedem Anbieter muss es dabei eine leistungsbezogen sicherstellende Vertragspflicht der Krankenkassen geben. Statt der Zulassung aller potentiellen Anbieter und ihrer kollektiven Regulierung im Nachhinein wir die Einführung des Vertragsprinzips zu mehr Qualität und Verlässlichkeit auf der Mikroebene der Versorgung führen und die Management-Unterstützung der Ärzte nachdrücklich verbessern können“. Dies unterstreicht der SVR-G, wenn er die Umsetzung „selektivvertraglichen Regelungen“ vorbehält (TZ 181), mit denen eine sektorenunabhängige Gebüh- renordnung (aus Relativgewichten) im Wettbewerb verpreist wird. Damit stellt er gerade den Schritt vor, „... der den Wettbewerb der Leistungserbringer um Patienten auch zu einem Wettbewerb der Krankenversicherer um gute Versorgung und damit letztlich auch um Versicherte werden lässt“ (TZ 183).

Dabei bietet es sich an, für eine hinreichende Übergangsphase eine einheitliche Vertragspflicht der Krankenkassen gegenüber 90 Prozent der zugelassenen Vertragsärzte, Institute und Krankenhäuser vorzugeben. Dadurch würde für die Patienten der jeweiligen Krankenkasse der offene individuelle Zugang zu einer möglichst hohen Anzahl von Spezialisten unabhängig von der „Rechtsform“ der Leistungserbringung sichergestellt und es entstünde gleichwohl ein spürbarer Wettbewerb um die beste Qualität der Versorgung. Über diese Vertragspflicht, die für die Krankenversicherungen jeweils einen gleichen Anteil an Leistungserbringern gilt, würde Aufsicht geführt. Im Bereich lebensbedrohlicher Erkrankungen mit geringem Zeitspielraum für Behandlungsentscheidungen müsste im begründeten Einzelfall ebenso wie im Hinblick auf die Teilnahme an kontrollierten klinischen Studien auch die Auswahl eines zugelassenen Leistungserbringers möglich sein, der nicht mit der entsprechenden Krankenkasse kontrahiert hat. Für unterversorgte Regionen könnten für die Krankenkassen besondere Kontrahierungsregelungen getroffen werden.

\section{Sektorenunabhängige Versorgung aus der Perspektive der Krankenhäuser}

Am Beispiel des „ambulanten Operierens“ wird die Notwendigkeit einheitlicher Rahmenbedingungen für Vertragsärzte und Krankenhäuser deutlich. Noch hat der Gesetzgeber gezögert, diesen Bereich in die Regelungssystematik des neuen \116b SGB V aufzunehmen. Aus gutem Grund - hätte doch ohne ein stimmiges Konzept zur Koordinierung eine sektorenübergreifende Budgetierung von Leistungsmengen ins Haus gestanden.

Die bestehende Regulierung krankt insbesondere an den Fehlanreizen der Vergütung. So ist es für die Krankenhäuser lukrativer, Kapazitäten im stationären Sektor mit Kurzzeitfällen auszulasten, als die Leistungen ambulant anzubieten. Im Sondergutachten 2012 wird vorgeschlagen, an das bestehende DRG-System anzuknüpfen und die Vergütung für die eigentliche vergleichbare Leistung durch Abzug von pauschalisierten Ist-Kosten für z. B. Übernachtung und Überwachung zu ermitteln. Damit wird auch die notwendige Abgrenzung zwischen vollstationären Operationen, Ein-Tages-Fällen und ambulanten Operationen gelöst. Auf der Grundlage einer solchen patientenbezogenen, aus der DRG-Systematik abgeleiteten Vergütung könnte sich der Wettbewerb zwischen Krankenhäusern, MVZs, Vertragsärzten und ambulanten Operationszentren auf der Basis von Selektivverträgen entwickeln.

\section{Entwicklung des ambulanten Vergütungssystems ${ }^{13}$}

Vom Grundsatz her sollten nicht einzelne ärztliche Verrichtungen und Diagnose- bzw. Therapieschritte das tragende Ordnungsprinzip bilden, sondern die indikations- und patientenbezogenen Behandlungsanlässe, von evidenzbasierten Leitlinien begleitet, klassifiziert nach Hauptdiagnosen, Nebendiagnosen und Schweregraden. Diesen Behandlungsanlässen wird der für sie regelmäßig anfallende Behandlungsbedarf zugeordnet, der den mit Hilfe von Kalkulationspraxen unabhängig festgestellten betriebswirtschaftlich erforderlichen Aufwand der Leistungen abbildet. Für Einzelleistungen, die im Einzelfall erforderlich, aber nicht regelmäßig einzukalkulieren sind, werden unter medizinischen qualitativen und erst in letzter Hinsicht ökonomischen Gesichtspunkten Abrechnungsvoraussetzungen formuliert. Insgesamt soll die Vergütung dem morbiditätsbedingten Behandlungsbedarf folgen, also den Bedarf des Patienten widerspiegeln. Bei der Weiterentwicklung der Vergütungsordnung darf sich dieses (Ziel-)Prinzip nicht wie bisher nur auf die Weiterentwicklung der Gesamtvergütungen beschränken, sondern muss sich auch so weit wie möglich in der be-

12 Vgl. Schönbach, K. H.: Sektorenübergreifende Sicherstellung mit Vertragspflicht der Krankenkassen, in: Gesundheitsund Sozialpolitik, S. $11-18$ sowie in: Jacobs, K., Schulze, S.: Sicherstellung der medizinischen Versorgung, Berlin 2011, S. $97 \mathrm{ff}$.

13 Vgl. hierzu auch den Bericht der FridrichEbert-Stiftung 2013, ebenda. Kapitel 3 , S. $17 \mathrm{ff}$. 
darfsgerechten Verteilung der Mittel an die Arztpraxen ausdrücken.

In diesem Sinne sollen die Abrechnungsbestimmungen und ökonomischen Anreize sich an der Förderungswürdigkeit der zu erbringenden Leistungen ausrichten. Bei den Hausärzten gilt es dabei insbesondere, der Versorgung chronisch kranker Patienten durch indikationsbezogene Zuschläge Rechnung zu tragen. In der spezialärztlichen Versorgung gilt es, komplexe Erkrankungen mit dokumentierter Einschlussdiagnose angemessen zu versorgen. Dabei muss der Zugang der Patienten zur Versorgung jeweils unabhängig von Einkommen und Sozialstatus erfolgen können. Allein pretial induzierte Leistungsanreize durch die offensichtlich nicht mehr reformierbare GOÄ, die einzelne Versichertengruppen bevorzugen, sind nicht weiter hinzunehmen. Sinnvoll wäre vielmehr ein einheitliches Vergütungssystem, das für Innovation offen ist, die einen Patientennutzen haben.

\section{Einheitliche Gebührenordnung GKV - PKV?}

Die PKV ist heute bereits Nutznießer des stationären Vergütungssystems mit DRG. Der Gesetzgeber hat ihr auch eingeräumt, an der frühen Nutzenbewertung neuer Arzneimittel in der GKV zu partizipieren. Wenn nun eine sektorenübergreifende ambulante Gebührenordnung entstehen soll, stellt sich auch unmittelbar die Frage nach einer Vereinheitlichung von EBM und GOÄ. Schließlich ist unverkennbar, dass das parallele Bestehen zweier Gebührenordnungen zu versorgungspolitisch unerwünschten Interaktionen führt, da die in der Zeit zurückgebliebene und fehlsteuernde private GOÄ ein Übermaß ärztlicher Behandlerzeit auf sich zieht. Das Gleiche gilt mithin für den stationären Sektor, wo neben der DRG-Vergütung im PKV Rahmen additiv GOÄ Ziffern abgerechnet werden dürfen - dieser Mechanismus ist in seiner Auswirkung nicht weit von den kritisierten Zielvereinbarungen auf Casemixpunkte entfernt. Zudem muss die Einführung einer neuen einheitlichen Gebührenordnung auch im Kontext der von der Politik diskutierten Einführung eines einheitlichen Krankenversicherungssystems betrachtet werden. Werden private und gesetzliche Versi- cherungsträger unter gleichen Systembedingungen tätig, wird ein unbestimmter Teil der bisherigen Bestandsversicherten in der angestammten privaten Krankenversicherung verbleiben wollen. Über den Erhalt des privaten Zusatzversicherungs-$$
\text { (1) }
$$$$
\text { Es ist unverkennbar, dass das }
$$$$
\text { allele Bestehen zweier }
$$$$
\text { Gebührenordnungen zu }
$$$$
\text { versorgungspolitisch uner- }
$$$$
\text { ünschten Interaktionen führt. }
$$

systems hinaus wird die private Vollversicherung mithin eine mehrjährige Übergangsphase mit sorgfältiger Gestaltung der Wahlmöglichkeiten für die bisherigen PKV-Versicherten benötigen. Eine versicherungsrechtlich bedingte Übergangsphase rechtfertigte jedoch nicht, die private Gebührenordnung Ärzte (GOÄ) mit ihren Fehlanreizen oder den bisherigen Einheitlichen Bewertungsmaßstab (EBM) mit seinen nicht nach Indikationen differenzierten, häufig am bloßen „Kontakt“ anknüpfenden Pauschalen fortzuführen und den Patienten eine neue, reformierte Gebührenordnung für ein gemeinsames Vergütungssystems vorzuenthalten. Vielmehr sollte dieses Vergütungssystem von Beginn an gelten und schrittweise weiterentwickelt werden, um die Vorteile für Ärzte und Patienten von Anfang zur Geltung zu bringen.

Eine einheitliche Gebührenordnung ist unter politischen Akzeptenzgesichtspunkten nur „in einem Schritt“ einführbar, wenn das Honorarvolumen zumindest aus dieser Maßnahme heraus nicht in Frage gestellt wird. Die Belastungen daraus sind den von Beteiligten zu tragen, die durch eine Abkehr von der GOÄ entlastet werden. Dies sind die PKV und die Beihilfeträger. Während damit für die PKV der Einbezug in den M-RSA verbunden wäre, wären die Beihilfeträger mit Zahlungen in den Gesundheitsfonds heranzuziehen.

\section{Vergütungssysteme: institutionelle Ausgestaltung}

Zur verlässlichen Realisierung einer morbiditätsorientierten Vergütungsordnung sind eine gleichfalls verlässliche Diagnosekodierung und damit eine Verpflichtung der Vertragsärzte auf die Anwendung ambulanter Kodier-Richt- linien unabdingbar. Die neue ambulante Vergütungsordnung ist wie oben angesprochen im Rahmen eines regelgebundenen und auf gesicherten Daten basierenden Prozesses auf betriebswirtschaftlicher Fundierung zu erstellen und mit dem stationären Vergütungssystem zu harmonisieren. Dies erfordert die Beauftragung eines fachlich kompetenten und unabhängigen Instituts. Die regelgebundene Erstellung der neuen Gebührenordnung sollte in der Kompetenz dieses Instituts liegen, das dabei an die Rahmenregelungen des Gesetzgebers und Richtlinien des Gemeinsamen Bundesausschusses gebunden ist. $\mathrm{Zu}$ diesem Zweck bietet sich ein Rückgriff auf das bisherige Institut des Bewertungsausschusses (InBA) und das Institut für das Entgeltsystem im Krankenhaus (InEK).

Das Scheitern von Vereinbarungen der Vertragspartner im Kompetenzbereich der Gebührenordnung für das erweiterte ambulante Vergütungssystem sollte eine Ersatzvornahme durch das BMG auslösen, wohingegen eine fehlende Einigung in Hinblick auf jährliche Preisvereinbarungen und Abrechnungsbestimmungen weiterhin eine Schiedsamtslösung oder die Entscheidung eines Schlichtungsgremiums nach sich ziehen sollte.

Morbiditätsbasierte Pauschalen hausärztlicher vs. fachärztlicher Bereich

Grundsätzlich sollte sich das Vergütungssystem auf morbiditätsbasierte Pauschalen stützen. Der bedarfsgerechte Differenzierungsgrad dieser Pauschalen wird allerdings je nach Versorgungsbereich unterschiedlich zu gestalten sein.

Im hausärztlichen Versorgungsbereich sollen die Pauschalen in Hinblick auf eine leitliniengerechte Versorgung der großen Volkskrankheiten definiert werden; in Hinblick auf die alternde Bevölkerung soll die Behandlung multimorbider Patienten aufwandsbezogen bei der Vergütung berücksichtigt werden. Das bloße Vorliegen von Diagnosen über mehrere Quartale kann Vergütungszuschläge nicht hinreichend begründen.

Dagegen sollte mit Blick auf das breitere Leistungsspektrum der Fachärzte und auf eine Harmonisierung zum DRG-System im fachärztlichen Bereich eine stärkere Ausdifferenzierung vorgenommen werden. Eine differenziertere Gestaltung der morbiditätsbasierten Pauschalen reduziert zwar die Anreize zur Risikoselektion, kann aber allein nicht Anreize zur Unterversorgung 
vermeiden. Insofern sind zusätzliche Einzelleistungen systematisch zu setzen und an medizinische Abrechnungsvoraussetzungen zu knüpfen. Damit werden die Behandlung auf der für das Patientenproblem angemessenen und erforderlichen Versorgungsstufe und eine innerärztlich sachgerechte Überweisungstätigkeit unterstützt. Darüber hinaus sind eine vertragliche Qualitätssicherung und zusätzliche qualitätssichernde Elemente der Vergütungsordnung notwendig.

Zudem werden für besonders förderungswürdige Einzelleistungen und besondere Auftragsleistungen weitere Elemente der Einzelleistungsvergütung sowie Komplexpauschalen erforderlich sein. Bei der Abrechnung der Gebührenordnungspositionen für Einzelleistungen sollte generell eine Differenzierung nach fixen und variablen Kostenanteilen vorgenommen werden. Der Fixkostenanteil soll in diesem Falle nur bis zu einem Höchstwert vergütet werden, so dass bei Leistungen über diesen Betrag hinaus nur der variable Kostenanteil vergütet wird. Hierüber werden sowohl die Leistungsgerechtigkeit des Vergütungssystems gegenüber den medizinischen Leistungserbringern gestärkt als auch Impulse zur nicht bedarfsgerechten Leistungsausdehnung aus dieser Vergütungsform abgeschwächt. Generell ist der Anteil der Leistungen in Einzelleistungsvergütung im Sinne der Vermeidung einer angebotsinduzierten Mengendynamik so klein wie möglich, jedoch so hoch wie zu einer angemessen differenzierten medizinischen Versorgung notwendig zu halten

\section{Zukünftige hausärztliche Vergütung}

Für den hausärztlichen Versorgungsauftrag stehen über Diagnostik und Therapie hinaus die Betreuung und Unterstützung der Patienten sowie die Koordination der hausärztlichen und fachärztlichen Versorgung im Mittelpunkt. Von daher sollten in der Vergütung Pauschalen dominieren, aber auch Anreize aufgenommen werden, die ggf. notwendige Technikausstattung vorzuhalten, um eine vorzeitige und verzichtbare Überweisungen zum Facharzt zu erübrigen. Die Vergütung des Hausarztes sollte sich in Zukunft aus folgenden Komponenten zusammensetzen:
- Altersadjustierte Fallpauschale,

- Morbiditätsbasierte Zusatzpauschalen für die leitliniengerechte Versorgung chronischer Erkrankungen wie z.B. Demenz, Diabetes, Hypertonie etc., gebunden an Parameter der Struktur- und Prozessqualität, unter Berücksichtigung von Koordinationstätigkeiten des Hausarztes mit ande-

Wird nun, wie oben gefordert, auch ambulant ein Leistungs-, Abrechnungsund Vergütungssystem eingeführt, bei dem ein inhaltlicher Zusammenhang zwischen patientenbezogenem Behandlungsanlass (Indikation) und Behandlungsinhalt (Leistung) und wo immer möglich Ergebnis (P4P Komponenten) dokumentiert wird, so kann die gewonnene Achtsamkeit für QualiFür die Beratung der Versicherten tätsaspekte auch ambulant haben die Qualitätssicherung mit Routinedaten sowie die Qualitätsvereinbarungen der Krankenkassen eine besondere Bedeutung.

ren ärztlichen und nichtärztlichen Leistungserbringern, ggfs. Zuschläge bei Multimorbidität

- Einzelleistungsvergütungen und Komplexpauschalen für besondere Leistungen

\section{Zukünftige fachärztliche Vergütung}

- Die fachärztliche Vergütung sollte je nach Versorgungsauftrag und Leistungen der betroffenen Facharztgruppen differenzieren. Strukturelemente dabei sind Jeweils angemessen differenzierte arztgruppenbezogene morbiditätsbasierte, ggf. nach Schweregraden und Nebendiagnosen justierte Pauschalen, gebunden an Parameter der Strukturund Prozessqualität und die leitliniengerechte Versorgung der entsprechenden Erkrankungen und für die (erweiterte) spezialärztliche Versorgung möglichst harmonisiert mit dem stationären DRG-Vergütungssystem

- Einzelleistungsvergütungen und Komplexpauschalen für besonders förderungswürdige sowie seltene Leistungen.

\section{Einführung von Qualitätsindikatoren}

Öffentlich gemachte Qualitätsberichte, externe Qualitätssicherung und internes Qualitätsmanagement haben in den vergangenen Jahren ambulant wie stationär die Aufmerksamkeit für Qualitätsaspekte der Versorgung maßgeblich erhöht. praxisgerecht genutzt werden, ohne jeweils auf zusätzlich zu erhebende Surrogat-Parameter zurückgreifen zu müssen.

Allein schon wegen der Trägervielfalt der ambulanten ärztlichen Versorgung scheint es in hohem Maße zweckmäßig, Qualitätsindikatoren in Richtlinien des Gemeinsamen Bundesausschusses zu fassen. Die Erfahrungen mit deutschen und internationalen Indikatorsystemen sind dazu vom IQWIG zusammenfassend zu bewerten. Dabei sind auch Kooperationsformen wie MVZ und Arztnetze zu berücksichtigen. Die Anwendung obliegt den jeweiligen Trägern der ambulanten Versorgung, die ihre jeweils vergleichbaren Berichte dem Gemeinsamen Bundesausschuss zuleiten und Erfahrungen austauschen. Auch die für die Versorgung zuständigen Aufsichtsbehörden der Länder sind berechtigt, entsprechende standardisierte Qualitätsberichte anzufordern.

Für die ambulante Behandlung muss die notwendige Transparenz durch einrichtungsbezogene Berichte, die überwiegend bürokratiearm aus Abrechnungsdaten erstellt werden können, geschaffen werden. Die Qualitätsberichte der Krankenhäuser sind mit Blick auf die neuen Herausforderungen anzupassen. Zweifellos kommt der Qualitätssicherung mit Routinedaten der Versorgung (QSR) künftig ebenso wie für Qualitätsvereinbarungen in Verträgen der Krankenkassen mit den Leistungserbringern auch für die Beratung der Versicherten eine besondere Bedeutung zu. Qualitätsindikatoren werden somit nicht nur Standards der Qualitätssicherung beschreiben, sondern auch wettbewerblich weiterentwickelt.

\section{Stationäre Sicherstellung}

Neben der notwendigen Verzahnung der Sektoren durch eine sektorenunabhän- 
gige Versorgung kommt die Gesundheitspolitik nicht umhin, die sektoralen Probleme schlüssig zu bearbeiten. Dies erfordert für die stationäre Versorgung das Zusammenwirken von Bund und Ländern. Der Bund kann zwar durch Gesundheitsfonds und Krankenhausvergütungsrecht die finanzwirtschaftlichen Bedingungen der Krankenhäuser maßgeblich gestalten, die Krankenhausplanung und -Zulassung ist jedoch den Ländern vorbehalten, die den Sicherstellungsauftrag haben. Zeiten, in denen Bund und Länder ihre Aufgaben an dieser Stelle harmonisch wahrgenommen haben, liegen zurück. Die stabile Phase der dualen Krankenhausfinanzierung seit 1972 endet zu Beginn der 1990er Jahre. Seither ziehen sich die Länder bis auf einen Anteil von inzwischen gerade 4,5 Prozent kontinuierlich aus der Krankenhausfinanzierung zurück. Seit Anfang der 2000er kommt hinzu, dass die Länder ihr Planungssystem unzureichend an das neue stationäre Vergütungssystem anpassen - es wird von dessen Dynamik geradezu überrollt.

\section{Produktivitätssprung durch DRGs treibt die Leistungsentwicklung}

Mit der bundesweiten Einführung des DRG-Systems ab dem Jahre 2003 wurde das einnahmeorientierte Fortschreiben historischer Krankenhausbudgets beendet. Im Ergebnis der neuen „leistungsorientierten Vergütung “ haben die
Krankenhäuser ihre Prozesse mit Hilfe der Kostenträgerrechnung transparent gestaltet und an den durchschnittlichen Fallkosten der Kalkulationskrankenhäuser orientiert. Werden dabei Kostenprobleme erkennbar, optimiert das jeweilige Krankenhaus seine internen Prozesse von der erforderlichen Verweildauer über die notwendige Diagnostik bis hin zur konkreten Kodierung. Somit hat das DRG-System einen beträchtlichen Innovationsschub ausgelöst, Optimierungsprozesse in Gang gesetzt und zu einem deutlichen Produktivitätssprung der deutschen Krankenhäuser beigetragen. ${ }^{14}$ Im Ergebnis hat sich die Zahl der vollstationären Pflegetage von 2003 bis 2010 um 11,5 Mio. Tage bzw. 7,6 Prozent verringert, obwohl im gleichen Zeitraum allein die altersbedingte Morbidität konservativ geschätzt um rund 8 Prozent zugenommen hat. Der parallele Fallzahlanstieg um rund 6,4 Prozent von 17,3 Mio. auf 18,5 Mio. Fälle steht dazu in keinem realen Widerspruch, da er in erster Linie Folge eines DRGinduzierten Fallsplittings ist und durch die Verkürzung der durchschnittlichen Verweildauer um 11,2 Prozent von 8,9 auf 7,9 Tage mehr als kompensiert wird. Weitere Steigerungen der Produktivität werden erwartet, zumal die Zunahme der Kurzlieger (Fälle von 1 bis zu 3 Tagen Verweildauer) von 30 auf 38 Prozent in dem Betrachtungszeitraum weiteres Optimierungspotential anzeigt. Diese Zunahme ist nur die Spitze eines für eine

\section{Abb. 3 Ausgabenentwicklung im Krankenhausbereich auf Basis der KJ1 ohne LKK}

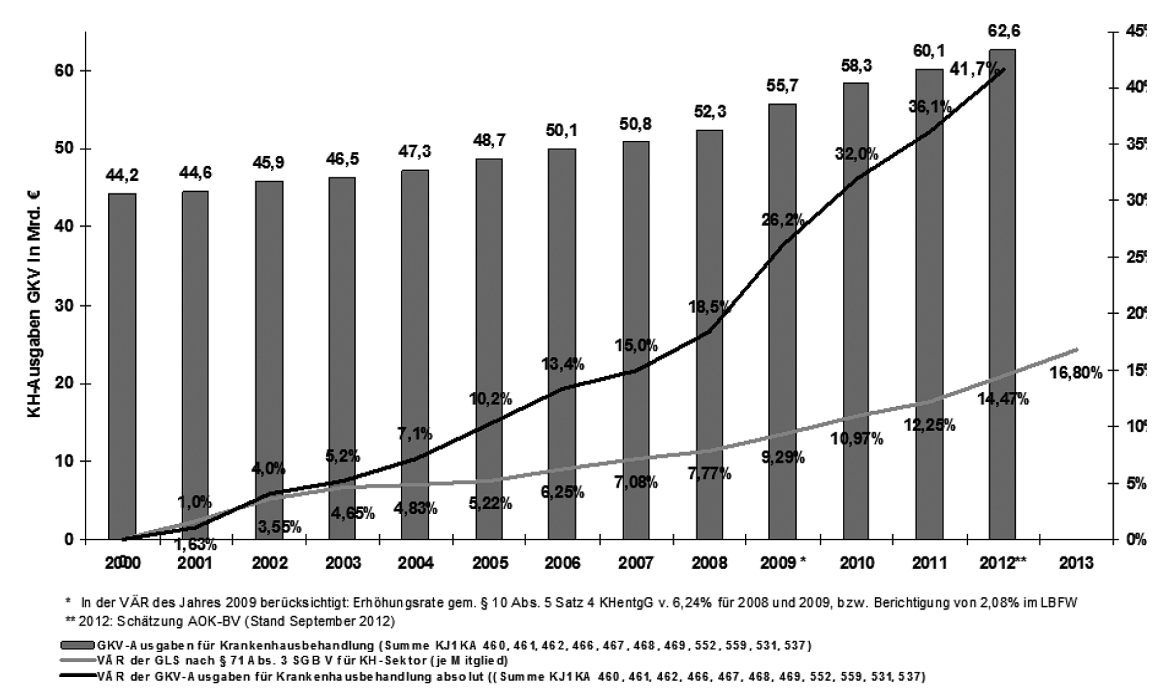

Quellen: eigene Darstellung; amtl. Statistik KJ1 GKV o LKK; VÄR GLS nach § 71 Abs. 3 SGB V destatis, Fachserie 12, Reihe 6.3
Ambulantisierung relevanten Eisberges, da die Krankenhäuser interessiert sein müssen, Abschläge bei Erreichen der unteren Grenzverweildauer regelhaft zu vermeiden. Werden die ordnungspolitischen Rahmenbedingungen zur Teilnahme der Krankenhäuser an einer sektorenunabhängigen ambulanten Versorgung verbessert, kann die Gesundheitspolitik zu weiteren Produktivitätssteigerungen der Krankenhäuser beitragen und darüber hinaus Anreize setzen, ohne die dem versorgungspolitisch fatalen Nachwuchsproblem für die hausärztliche Versorgung nicht begegnet werden kann.

\section{Finanzierungsstrukturen und Kapazitätsauslastung}

Die Ausgaben der GKV für stationäre Leistungen sind von rund $44 \mathrm{Mrd}$. Euro im Jahre 2000 auf rund 60 Mrd. Euro im Jahre 2011 angestiegen. Trotz kostendämpfender Eingriffe des Gesetzgebers liegen die Zuwachsraten der Ausgaben weiterhin deutlich über der Veränderungsrate der beitragspflichtigen Einnahmen ( $\int 71$ SGB V). Auch von hieraus bleiben Steuerzuschüsse zum Gesundheitsfonds angezeigt, wenngleich die Ursachen inzwischen hinterfragt werden. Nunmehr erkennt auch die Gesundheitspolitik, dass die Entwicklung der stationären Ausgaben primär von der Mengenentwicklung bestimmt ist. Symptome wie etwa Mengen-induzierende „Zielvereinbarungen“ der Krankenhäuser mit leitenden Ärzten sollen „gesetzlich verboten“ werden.

- In der Tat erweist sich aufgrund der DRG-Begleitforschung nur ein Drittel der stationären Mehrleistungen durch demographische Änderungen erklärbar. Zwischen 2006 und 2010 sind die DRG-Leistungsmengen (Casemix-Punkte) im Durchschnitt um 3,1 Prozent jährlich gestiegen, davon die Fallzahlen um 2 Prozent pro Jahr. Über den medizinischen Fortschritt und die Zunahme von Behandlungsmöglichkeiten hinaus ist inzwischen eine Leistungsvermehrung über das medizinisch bedarfsgerechte Maß hinaus zu beobachten, weil:

14 Vgl. IGES: DRG-Begleitforschung gemöß $\S 17 \mathrm{~b}$ Abs. $8 \mathrm{KHG}$ - Endbericht des dritten Forschungszyklus (2008 bis 2010, Untersuchung im Auftrag des deutschen DRGInstituts (InEK), Berlin, März 2013. 
- das fallpauschalierte, leistungs(mengen)orientierte DRG-System Anreize zur Erhöhung der Leistungszahlen beinhaltet, weshalb häufig monetäre Prämien als variable Gehaltsbestandteile für Krankenhausärzte an das Erreichen besonderer Fallzahlsteigerungen geknüpft werden.

v durch eine primär „nachvollziehende“ Krankenhausplanung Überkapazitäten bestätigt werden, die wiederum alle Krankenhäuser zu einer „ökonomischen“ Belegungspraxis gerade zur Deckung der Vorhaltekosten zwingen, während von der politischen Seite her Marktaustrittsbarrieren bestehen.

- die nicht substanzgerechte Investitionskostenfinanzierung der Bundesländer die Krankenhäuser darauf verweist, Investitionen weitgehend über die Steigerung der Leistungsmengen zu refinanzieren. ${ }^{15}$

Für die Krankenkassen besteht gegenüber allen (Plan-) Krankenhäusern ein Vertragszwang, obschon die Bettenauslastung von immerhin noch durchschnittlich 82 Prozent im Jahre 2000 auf 75 Prozent im Jahre 2004 gesunken ist und seither bei rund 77 Prozent (2008) verbleibt. ${ }^{16}$ Dabei sehen die Krankenhauspläne zahlreicher Bundesländer eine „Mindestauslastung“ von 85 Prozent bei somatischen Krankenhäusern als sachgerechte Untergrenze an. ${ }^{17}$ Konserviert wird damit auch ein Strukturproblem, auf das etwa in den Ratings des RWI hingewiesen wird. ${ }^{18}$ Moderne Krankenhäuser sollten eine Mindestgröße von rund 500 Betten aufweisen, um ein akzeptables Verhältnis von Gemeinkosten zu variablen Fallkosten erzielen zu können. Im Jahre 2011 lagen jedoch 68 Prozent aller deutschen Krankenhäuser (inklusive der psychiatrischen Häuser) unterhalb von 300 Betten. Bezogen auf die gesamte Bettenkapazität beträgt der Anteil immerhin knapp 33 Prozent. Auch wenn relativierend anzumerken ist, dass die betriebswirtschaftlich genannte Bettengrenze für spezialisierte Fachkliniken weniger bedeutsam ist, ist das Strukturproblem erkennbar. Und es bleibt beileibe nicht auf die betriebswirtschaftlichen Aspekte begrenzt. Nicht nur die von der Deutschen Krebsgesellschaft zertifizierten onkologischen Zentren verweisen auf Qualitätsvorteile für die Patienten. Vielmehr unterscheiden sich
Behandlungsergebnisse vielfach signifikant nach an größeren Krankenhäusern gelegenen Zentren und sonstigen Standorten mit geringerer Spezialisierung (z.B. auch Leistungen der elektiven Endoprothetik im QSR Verfahren). Gerade hier aber findet zurzeit geradezu ein Run auf hochspezialisierte und hoch vergütete DRGs im elektiven Bereich statt (Wirbelsäulenoperationen, kardiologische Eingriffe als Beispiel): Durch den allgemeinen Produktivitätssprung aufgrund der DRG-Einführung aufgedeckte Überkapazitäten werden somit weithin unter dem Anreiz von Zielvereinbarungen für die Expansion elektiver Eingriffe verwendet, ohne dass der Patientenschutz an oberster Stelle steht.

Insbesondere wenn qualitätssichernden Mindestmengen der Selbstverwaltung unter rechtlichen Aspekten Grenzen gezogen werden, die nicht dem Patientenschutz genügen, ist die Krankenhausplanung auch unter Qualitätsaspekten zu gestalten verpflichtet. So sind nicht nur qualitätssichernde Mindestgrößen neonatologischer Intensivstationen ungesichert. Erst angesichts medizinischer Manipulationen bei der Organverteilung wird auch zur Kenntnis genommen, dass an über 50 deutschen Kliniken häufig in erschreckend geringen Fallzahlen transplantationschirurgische Eingriffe stattfinden. Ebenso muss gefragt werden, warum in Deutschland 25 von 32 Universitätskliniken Kinderherzchirurgie betreiben. Die Schweden haben es in einem Land mit großer Fläche geschafft, diese Chirurgie in zwei Zentren zu konzentrieren, was zwar häufig lange Anfahrtswege, dafür aber aufgrund größerer Zahlen hinreichende Erfahrung bei der komplizierten Operation bedeutet. Auslöser für diese Konzentration war gerade auch der Elternwunsch herzkranker Kinder.

Den deutschen Planungsbehörden fehlt aber für eine medizinisch-epidemiologische Gestaltung zum einen der politische Auftrag. Vielfach werden Krankenhäuser politisch als Bestandteil der regionalen Wirtschaftsförderung betrachtet: Finden Abwägungen dieser Art etwa für ländliche Regionen noch Verständnis und sind in Richtung auf Notfallzentren bzw. Rettungsmittel (Hubschrauber) sowie eine Umwandlung in MVZ aufzulösen, gefährden
Mehrfachstrukturen für Verdichtungsräume aufgrund ihrer ökonomischen Anreize die Beachtung medizinischer Indikationsgrenzen und den notwendigen Patientenschutz. Die Gefahr der Überbehandlung stellt sich nicht erst seit der Diskussion über Zielvereinbarungen leitender Ärzte. Auch die Deutsche Gesellschaft für Chirurgie räumt entsprechende Probleme aufgrund von Überkapazitäten in Deutschland ein. Zum anderen mangelt es den Planungsbehörden schlicht an hinreichenden rechtlichen Grundlagen, um Entscheidungen aus komplexen Zusammenhängen problemgerecht herzuleiten, gesundheitspolitisch abzustimmen und so rechtlich fundiert abzusichern, dass Entscheidungen in zügige und unmittelbar umsetzbare Verwaltungsakte münden können. Sich jahrelang hinziehende und unklare Prozesse waren bisher oft Folge von Bemühungen der Länder, eine Aufnahme in den Krankenhausplan in Frage zu stellen.

\section{Weiterentwicklung der Krankenhausplanung}

Die bestehende Krankenhauslandschaft beruht mehr oder weniger (in den neuen Ländern) auf dem althergebrachten Standortangebot, das bei seiner historischen Etablierung von inzwischen weithin abgelegten medizinischen und versorgungstechnischen Grundsätzen geprägt war: So war die Medizin in den Gründerjahren weit weniger spezialisiert, weniger geräteaufwendig, eher pflege- als funktionsorientiert und das Krankenhaus spielte bei der Notfallerstversorgung eine wichtige, häufig entscheidende Rolle. Diese Aufgabe der Erstversorgung übernehmen heute die Notarztsysteme. Mithin ist die be-

15 Vgl. Malzahn, Jürgen; Wehner, Christian: Zur Lage der Investitionsfinanzierung der Krankenhäuser - Bestandsaufnahme und Reformbedarf; in: Klauber, Jürgen; Geraedts, Max; Friedrich, Jörg (Hrsg.): Krankenhaus-Report 2010 - Schwerpunkt: Krankenhausversorgung in der Krise, Stuttgart 2010, S. 107-125.

16 Vgl. Statistisches Bundesamt: Gesundheit - Grunddaten der Krankenhäuser 2011, Fachserie 12 Reihe 6.1.1; Wiesbaden, 18.10.2012.

17 Vgl. Weigl P.; Pressekonferenz „Krankenhauslandschaft im Umbruch" - Statement von Vizepräsident Peter Weigl. Berlin, 10. Dezember 2008.

$18 \mathrm{Vgl}$. Augurzky et al.: Krankenhaus Rating Report 2012 - Krankenhausversorgung am Wendepunkt?; Heidelberg 2012. 
stehende Krankenhausdichte in erster Linie historisch mit der Notwendigkeit der schnellen medizinischen Notfallversorgung erklärbar.

Die Bundesländer müssen nach $\mathbb{} 6$ KHG zur Gewährleistung einer bedarfsgerechten Versorgung der Bevölkerung Krankenhauspläne aufstellen, wobei die Einzelheiten in Krankenhausgesetzen der jeweiligen Bundesländer geregelt werden. Zahl und Art der Krankenhausbetten orientieren sich an Kennzahlen wie der Bevölkerungszahl, der Verweildauer im Krankenhaus und der Bettenauslastung. Damit wird der Bedarf der Vergangenheit normativ festgelegt und fortgeschrieben, ohne den tatsächlichen Versorgungsbedarf zu berücksichtigen.

Zwar unternehmen auch einzelne Bundesländer Anstrengungen, die Krankenausplanung zu qualifizieren und Qualitätsindikatoren ${ }^{19}$ in den Krankenhausplan aufzunehmen. Die allgemeine Entwicklung und der Bedarf, mit den Krankenhäusern über die Landeskrankenhausgesellschaften hinaus zu übergreifenden Vereinbarungen zu kommen, lassen allerdings eine stärkere bundesweite Regulierungsvorgabe des G-BA als geraten erscheinen. Sie bietet sich auch an, wenn sich die Bundesländer aufmachen, ihre regionale Verantwortung für den ambulanten und sektorenunabhängigen Teil der Versorgung zu stärken ( $\sqrt{90 a}$ SGB V).

\section{Grundsätze einer gestuften Planung}

Für den stationären Sektor wird ein neues, gestuftes System der Krankenhausplanung benötigt. Dem Grundsatz der Einheitlichkeit der Lebensverhältnisse folgend und weil der Gesundheitsfonds eine Finanzierung nach bundeseinheitlichen Maßstäben sicherstellt, sollten die stationären Angebotsstrukturen als Zielgröße mit Populationsbezug bundesweit festgelegt und aktualisiert werden. Allein schon aufgrund des leistungsbezogenen Vergütungssystems sind auch die Angebotsstrukturen leistungsbezogen zu formulieren. Wie die Angebotsstrukturen krankenhausbezogen konkret zugeschnitten werden bleibt dezentralen Entscheidungen überlassen. Je nach spezialisierungsnotwendiger Mindestgröße sind zudem bundeseinheitlich die Planungsmaßstäbe differenzierende Zeitspannen der Erreichbarkeit als Orientierungsgröße festzulegen. Diese Aufgabe sollte der Gesetzgeber nach wissenschaftlicher Aufbereitung dem Gemeinsamen Bundesausschuss zur Beratung für Richtlinien übertragen.

Die eigentliche Sicherstellung der medizinischen Versorgung ist ausgehend vom Sozialstaatsprinzip eine Aufgabe öffentlicher Daseinsvorsorge der Länder, die auf dem Sozialstaatsprinzip beruht. Sicherzustellen ist eine bedarfsorientierte Versorgung, wobei die Selbstverwaltung gemeinsam mit den Ländern die Raumplanung erstellt. Über die Richtlinien des Bundesausschusses hinaus hat das jeweilige Land dabei regionale Besonderheiten aufgrund der regionalen Morbidität und komplementärer Angebotsstrukturen sowie der regionalen Bevölkerungsdichte und Verkehrsinfrastrukturen

\section{Kollektive Budgets und} mangelnde Qualitätsanreize sind Folge des Kontrahierungszwangs.

zu berücksichtigen. Für eine stationäre Raumplanung sind folgende Bereiche zu unterscheiden:

1. Notfallversorgung mit einer qualitativ definierten Erreichbarkeit: Hier bietet sich der Rückgriff auf die in den Rettungsdienstgesetzen der Länder festgelegten Hilfsfristen an. So wird im Hessischen Krankenhausrahmenplan die Notfallversorgung als enge Verzahnung zwischen Rettungsdienst und Krankenhaus beschrieben. Die Regelentfernung zwischen Notfallort und Krankenhaus sollte 15 bis $25 \mathrm{~km}$ betragen. Somit ergeben sich rechnerisch ca. $35 \mathrm{~km}$ als Entfernung zwischen Notfallkrankenhäusern.

2. Medizinische Hochschulstandorte der Länder: Auch wenn Uniklinika zum Teil überwiegend Krankheitsbilder behandeln, die mit geringerem Ressourceneinsatz ebenso gut und wohnortnäher von Krankenhäusern der Grundund Regelversorgung versorgt werden könnten, haben die Länder für diese Krankenhäuser eine besondere Verantwortung. Daher werden die Universitätskrankenhäuser von allen folgenden Regelungen ausgenommen und nur bei der Planung der Notfallversorgung berücksichtigt.

3. Medizinische Leistungsbereiche für einheitliche und gemeinsame Versorgungsverträge: Neben der Notfallversorgung ist es für bestimmte Krankheitsgebiete sinnvoll, wenn sie von allen grundsätzlich geeigneten Krankenhäusern unter Beibehaltung einer vollständigen Vertragspflicht der Krankenkassen erbracht werden können. Allerdings sollten hier Qualitätsvorgaben des G-BA oder anderer maßgeblicher Institutionen die Eignung mit ausschließendem Charakter bei Nichterfüllung untermauern, damit die Qualitätsvorgaben angemessen berücksichtigt werden.

Es handelt sich beispielsweise um Leistungen, für die hohe Investitionen und spezielle regional zu planende infrastrukturelle Voraussetzungen von Relevanz sind. Dies gilt für Bereiche wie die Onkologische Versorgung. Hier sollten alle nach den Kriterien der Deutschen Krebsgesellschaft zertifizierten Krankenhäuser auch an der stationären Krankenversorgung teilnehmen können. $\mathrm{Zu}$ überlegen ist auch, ob bei weiteren Krankheiten (z.B. Demenzerkrankungen), bei denen ein aktives Wahlverhalten von Patienten eingeschränkt sein kann, auch zukünftig alle Krankenhäuser mit entsprechender Ausstattungen bzw. Fachabteilungen grundsätzlich an der Versorgung teilnehmen sollten. Die Erstellung einer abschließenden und konkreten Krankheitsliste ist jedoch unerlässlich, damit die Vertragspflichten zwischen diesem durch einheitliche Qualitätsanforderungen und Verträge geregelten Versorgungsbereich und dem verbleibenden Wettbewerbsbereich rechtssicher abgegrenzt sind.

4. Medizinische Leistungsbereiche für Einzelverträge unter Berücksichtigung der Qualität: Die verbleibenden Leistungen werden über Einzelverträge zwischen Krankenhäusern und Krankenkassen vertraglich vereinbart. Dabei sind die Krankenkassen zunächst

19 Vgl. Jochen Metzner: Krankenhausplanung - was ist das und wie kann man Qualitätskriterien einbauen? In: Gesundheits- und Sozialpolitik, Heft 4 in 2013, Seite 25 ff. und Elke Huster-Nowack, Matthias Gruhl: Krankenhausplanung zwischen Versorgung und Wettbewerb, ebenda, Seite $32 \mathrm{ff}$. 
verpflichtet mit 90 Prozent der Krankenhäuser unter Beachtung von Qualitätskriterien Versorgungsverträge zu schließen. Für die Vergabe steht dabei eine Bewertung nach Qualitätskriterien im Vordergrund. Zweifellos aber sollte der Vertragskorridor auch einen Preiswettbewerb der Krankenhäuser ermöglichen, bei dem die DRGs i.V.m. dem jeweiligen Landesbasisfallwert Preisobergrenzen bilden. Dieses Vorgehen hat den Zweck, die Mengenentwicklung der letzten Jahre auf ein medizinisch erforderliches Maß zu reduzieren. Davon sind insbesondere die Leistungsbereiche betroffen, in denen die höchsten Steigerungsraten zu verzeichnen sind, ohne dass rationale medizinische Gründe vorgebracht werden können. Die Länder haben über diesen Vergabeprozess die Aufsicht.

Aus der Komplexität der Parameter ergibt sich, dass das Land im Hinblick auf eine Krankenhauslandschaft unter dynamischem Wettbewerb keine Standortentscheidungen treffen soll. Vielmehr erstellt das Land jenseits der Universitätskliniken unter (2.) eine landesbezogene Raumplanung, deren Erfüllung es aufsichtsrechtlich sicherstellt.

Es ist in Studien vielfach dargelegt worden, dass der Übergang zum Einzelvertragsprinzip Wegezeiten der Patienten nur geringfügig erhöht. ${ }^{20}$ Auch ist der Anreizeffekt für Qualität, Service und Wirtschaftlichkeit ungleich bedeutsamer als ein ungewisser Korrektureffekt auf Überkapazitäten. Daher wird vorgeschlagen, auch im Einzelvertragskonzept eine einheitliche Vertragspflicht für alle Krankenkassen vorzusehen, die 90 Prozent der verfügbaren Angebote einschließt. Dadurch wird der offene Zugang des Patienten zu verfügbaren Angeboten sichergestellt und der Korrektureffekt des Wettbewerbs auf die Grenzen beschränkt, die er tatsächlich auch erreichen soll.

Insoweit sich die neue „Raumplanung “ tatsächlich von der bisherigen „Standortplanung“ entfernt und eine Vertragspflicht der Krankenkassen klar abgegrenzte Freistellungen aufweist, ist ein Übergang zur monistischen Krankenhausfinanzierung mit Ausnahme der medizinischen Hochschulstandorte der Länder ordnungspolitisch geboten. Entsprechende Mittel für die Investitionsfinanzierung sind dann in den Gesundheitsfonds einzustellen.

\section{Fazit}

Die Anpassung der institutionellen Beziehungen und Regelungen innerhalb des Gesundheitssystems sind eine Daueraufgabe, bei der zahlreiche Verflechtungen und externe Effekte berücksichtigt werden müssen. Deutschland hat inzwischen ein hochstehendes leistungsbezogenes Vergütungssystem für Krankenhäuser, das die althergebrachte Fortschreibungskultur der betten- und abteilungsbezogenen Krankenhausplanung schlicht überfordert. Die Folge sind Anreize zu einer inflationären Leistungserbringung, die mittlerweile die ethischen Grundlagen des Medizinbetriebs tangiert. Wir brauchen daher eine populationsbezogene, medizinisch strukturierte und begründete Krankenhausplanung, die sektorenübergreifend erstreckt wird, um auch die spezialärztliche Versorgung abzubilden. Dies ermöglicht, der medizinisch de facto fortgeschrittenen Ambulantisierung auch institutionell Raum zu geben und stellt die Beteiligten vor die Herausforderung, hinreichende Anreize für einen Qualitätswettbewerb zu organisieren. „Formale Zulassung“, „landesweite Budgets“ und „Verfall der Vergütungen“ angesichts medizinisch nicht begründeter Mengenentwicklungen dürfen für dieses Herzstück der ambulanten Versorgung nicht in Kauf genommen werden. Der vorgeschlagene Weg, dies zu ermöglichen, sind qualitätsorientierte Ausschreibungen auf Ebene der Einzelkasse. Werden alle Krankenkassen verpflichtet, 90 Prozent der verfügbaren Leistungsangebote unter Qualitätsgesichtspunkten auszuwählen, entsteht ein hinreichender Qualitätswettbewerb. Gleichzeitig behalten die Patienten sehr weite Auswahlmöglichkeiten im Rahmen eines vielfältigen Angebotes. Dieses Vertragsprinzip ist ebenso für die planbaren stationären Leistungen der Krankenhäuser anwendbar. Die von einer bedarfsorientierten neuen Krankenhausplanung ausgehenden Ziele der Kapazitätsanpassung können somit in einem einzelvertraglichen Korridor umgesetzt werden, ohne dass staatliche „ja/ nein“-Entscheidungen erforderlich würden. Dies erlaubt den Krankenhäusern auch Anpassungsreaktionen und belässt Zeiträume der Umstrukturierung.
Die politische Ermöglichungen eines solchen Modernisierungsprozesses reduziert Überkapazitäten im stationären Bereich, erlaubt eine Entlastung der fachärztlichen Versorgung ambulant und gibt damit auch Anreize, mit denen die hausärztliche Tätigkeit wieder vergleichsweise attraktiv wird bzw. mehr Interessenten findet. Dies entspricht der Entwicklung des Bedarfs bzw. verbessert die Allokation im Gesundheitswesen. Störungen hierfür gehen immer noch von der Dualität der privaten (die eine staatliche ist) und gesetzlichen (die eine vertragliche ist) Gebührenordnung für PKV und GKV aus. Sie verzerren ohne Rechtfertigung in der ärztlichen Versorgung die für den Arzt relevanten Preisstrukturen: $\mathrm{Zu}$ kunftsweisend ist es, die Preisstrukturen ambulant wie stationär an dem patientenbezogenen Bedarf auszurichten, das
Zukunftsweisend ist es, die Preisstrukturen ambulant wie stationär an dem patientenbezogenen Bedarf uszurichten.

heißt an der Morbidität: Dies erfordert den Indikationsbezug der zu vergütenden Leistung. Und zukunftsweisend ist es, die Leistungen - über notwendige Einzelleistungen hinaus - als indikationsbezogene Pauschale betriebswirtschaftlich fundiert mit Hilfe von Kalkulationspraxen zu kalkulieren: Dies erfordert ein unabhängiges Institut für die Bewertung ärztlicher Leistungen wie es für die stationären Entgelte bereits besteht. Heute ist lediglich die Mittelaufbringung bei der Kassenärztlichen Vereinigung morbiditätsorientiert, während die Verteilung der Honorare wechselnden Mehrheitsverhältnissen der Arztgruppen in der KV und ihrer Verteilungskämpfe unterliegt. Niemand würde ein solches System, das auf die Notverordnungen der 30er Jahre des vorigen Jahrhunderts zurückgeht, den Ärzten und Patienten zur Abstimmung stellen: Grund genug also, die Versorgungsstrukturen zu modernisieren.

20 Göbel, Thomas; Wolff, Johannes: Direktverträge für stationäre Leistungen - Chance für mehr Qualität und Wirtschaftlichkeit im Krankenhaussektor; in: Klauber, Jürgen; Geraedts, Max; Friedrich, Jörg (Hrsg.): Krankenhaus-Report 2012 - Schwerpunkt: Regionalität, Stuttgart 2012, S. 123-147. 